\title{
TEMPERATURE MAPPING RESULTS ON THE HIGH-FIELD Q-SLOPE OF 1500 MHz SINGLE CELL SUPERCONDUCTING RADIOFREQUENCY CAVITIES BAKED IN-SITU AT $400 \mathrm{C}^{*}$
}

\begin{abstract}
The heat treatment of a niobium cavity between $100^{\circ} \mathrm{C}$ - $120^{\circ} \mathrm{C}$ for 48 hours substantially improves cavity performance, presumably by healing the nature of the oxidemetal interface, although the nature of the healing is not yet understood. The heat treatment at higher temperatures is found to deteriorate the performance. Our tests on 1500 MHz single cell cavities are always equipped with a temperature mapping system [1] consisting of 700 thermometers. The effect of heat treatment at various temperatures has been studied in detail using the temperature mapping system. In this article we report on several interesting findings from studies of a $400^{\circ} \mathrm{C}$ heat treatment.
\end{abstract}

G. Eremeev ${ }^{\dagger}$, H. Padamsee, CLASSE, Cornell University, Ithaca, NY, 14853, USA

\section{INTRODUCTION}

Two phenomena, high field Q-slope and residual resistance, are limiting factors for superconducting cavities. The origin of these phenomena is not yet fully understood. In the early 1990's F. Palmer carried out experiments on $8400 \mathrm{MHz}$ niobium oxide-free cavities, in order to study effect of niobium oxide on a low field surface resistance of niobium cavities [2]. In order to remove oxide he heat treated cavities at $1100^{\circ} \mathrm{C}$. At that time most cavities were limited by field emission, so the effect of such treatment on a high field performance was not studied. We decided to apply a high temperature heat treatment to niobium cavity to study an effect of niobium oxides on high field performance.

The interest in the high field Q-slope and in the heat treatment effect triggered surface studies on niobium samples before and after heat treatment at different temperatures. XPS studies have shown that niobium oxide can be destroyed at temperatures as low as $250^{\circ} \mathrm{C}$ [3]. Other studies suggested that temperatures around $400^{\circ} \mathrm{C}[4]$ are needed to remove the oxide. Also it was shown that oxidefree surface stays oxide-free for hours in vacuum. In this article we report on results of superconducting niobium cavities performance at high fields after $400{ }^{\circ} \mathrm{C}$ heat treatment.

\section{EXPERIMENTS}

The cavity was $1.5 \mathrm{GHz}$ elliptical cavity with $1 \mathrm{~mm}$ grain size. It was made of $300 \mathrm{RRR}$ niobium sheet of $3 \mathrm{~mm}$ thickness. The half-cells, shaped by deep drawing, were postpurified at $1300^{\circ} \mathrm{C}$ for 2 hours and then at $1200^{\circ} \mathrm{C}$ for 4

\footnotetext{
* Work supported by NSF

$\dagger$ gve2@cornell.edu
}

hours. The half-cells were vertically electropolished [5] before welding. After welding the cavity was vertically electropolished for about $100 \mu \mathrm{m}$. After electropolishing the cavity was chemically treated with $\mathrm{BCP}\left(\mathrm{HF}: \mathrm{HNO}_{3}: \mathrm{H}_{3} \mathrm{PO}_{4}\right.$ - 1:1:2) for $10 \sec ($ flash $\mathrm{BCP}$ ). The temperature of the solution was kept below $15^{\circ} \mathrm{C}$. After the chemical treatment the cavity was rinsed with ultra pure water and transferred to the clean room of class 100 . In the clean room the cavity was rinsed for two hours with high purity water under 1000 psi pressure. Then the cavity was assembled on the 1500 $\mathrm{MHz}$ test stand in the clean room. After assembly the cavity was lowered into the dewar and was tested at the bath temperature of $1.5^{0} \mathrm{~K}$ at low fields and increased to $1.7^{0} \mathrm{~K}$ at high fields.

Next the cavity was heat treated at $400^{\circ} \mathrm{C}$ for 2 hours. For the heat treatment a steel box was made, put around the cavity and pressurized with argon during the heat treatment, to avoid oxidation of the outer surface of the cavity. The inner surface of the cavity was kept under vacuum. The heating elements were two $1.5 \mathrm{~kW}$ band heaters placed on copper rings, which were shaped to follow the curvature of the outer surface of the cavity. Copper foils were used to improve the temperature distribution. Only the cell itself was heat treated, the rest of the test stand was at a room temperature because of water cooling clamps on beam pipes. The pressure during the heat treatment was $4.5 \cdot 10^{-7}$ Torr. The main constituents were water $\left(2 \cdot 10^{-7}\right.$ Torr) and $\mathrm{CO}\left(3 \cdot 10^{-8}\right.$ Torr $)$ as was indicated by residual gas analyzer. The oxygen partial pressure was $8 \cdot 10^{-11}$ Torr. The cavity was kept at above $400^{\circ} \mathrm{C}$ for two hours. After the heat treatment the cavity was tested again at the bath temperature of $1.5^{0} \mathrm{~K}$ at low fields and increased to $1.7^{0} \mathrm{~K}$ at high fields.

After the second test the cavity was warmed up to room temperature and pressurized with dry air (VOC Zero Grade Air from Airgas) to 2.4 Torr for 5 minutes, to form an oxide layer. Then it was pumped down to $10^{-7}$ Torr and tested at the bath temperature of $1.5^{0} \mathrm{~K}$ at low fields and increased to $1.7^{0} \mathrm{~K}$ at high fields.

After the third test the cavity was heat treated with the same setup as before at above $400^{\circ} \mathrm{C}$ for two hours, to remove the oxide layer again. After the heat treatment it was tested at the bath temperature of $1.5^{\circ} \mathrm{K}$ at low fields and increased to $1.7^{0} \mathrm{~K}$ at high fields.

\section{RESULTS}

In Fig. 1 we present the quality factor of the cavity as a function of peak magnetic field for all four tests. Con- 


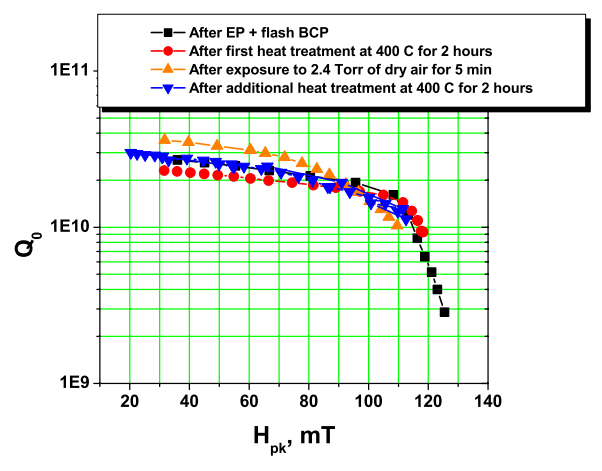

Figure 1: Quality factor as function of field before and after heat treatment.

sidering RF performance of TESLA-like superconducting cavities, one distinguishes three different types of behavior, Q-slopes: the low field Q-slope, which is sometimes observed for peak surface magnetic fields below $20 \mathrm{mT}$, the medium field Q-slope, a weak degradation of the quality factor for peak surface magnetic fields between $20 \mathrm{mT}$ and $100 \mathrm{mT}$, the high field Q-slope, a strong degradation of the quality factor above $100 \mathrm{mT}$. We didn't take data points below $20 \mathrm{mT}$, so we didn't see a low field Q-slope in any of our tests. To quantify a medium field Q-slope we used $\frac{G}{Q}=R_{s}\left(1+\gamma\left(\frac{H}{H c}\right)^{2}\right)$, where $R_{s}$ is a fitting parameter and can be interpreted as a mean surface resistance, $\gamma$ is fitting parameter, $\mathrm{Hc}=2000 \mathrm{mT}$ is the critical field of niobium, to fit the data for peak surface magnetic fields from $20 \mathrm{mT}$ to $100 \mathrm{mT}$.

In the first test $R_{s}=8.88 \pm 0.03 \mathrm{nOhm}$ and $\gamma=$ $2.14 \pm 0.03$. Above $110 \mathrm{mT}$ we observed high field Qslope. The highest peak magnetic field was $123 \mathrm{mT}$ and at this point we were limited by available RF power. The temperature map taken above $110 \mathrm{mT}$ showed three hottest regions on the bottom half-cell, Fig. 2. All temperature sensors in the high magnetic field's region showed a nonquadratic increase in heating, the high field Q-slope, above $100 \mathrm{mT}$. The typical temperature sensor's reading is presented at Fig. 3(squares).

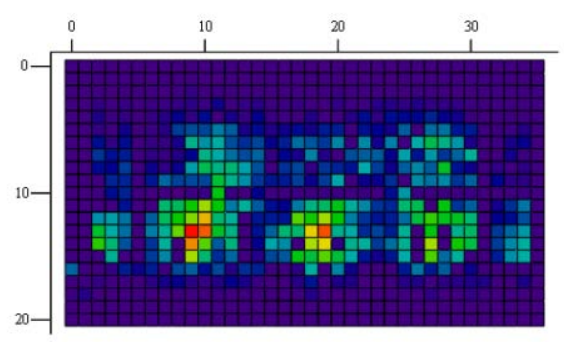

Figure 2: Temperature map for the first test at $\mathrm{H}_{\text {peak }}=123$ $\mathrm{mT}$.

In the second test $R_{s}=10.67 \pm 0.03 \mathrm{nOhm}$ and $\gamma=$
$1.72 \pm 0.03$. Again above $110 \mathrm{mT}$ we observed a high field Q-slope. The highest peak magnetic field was $117 \mathrm{mT}$ and the cavity was limited by thermal breakdown. The temperature map showed four new hot regions on the bottom half-cell at highest field, Fig. 4. Three of these regions, as the analysis of the temperature sensors' data showed, had quadratic losses at all fields, but they had about a ten times higher surface resistance than other areas, Fig. 5(circles). These regions with high residual resistance covered about 15 percent of cavity surface. The thermal breakdown was associated with one of these regions. The rest of the temperature sensors showed a behavior typical for the high field Q-slope, Fig. 3(circles).

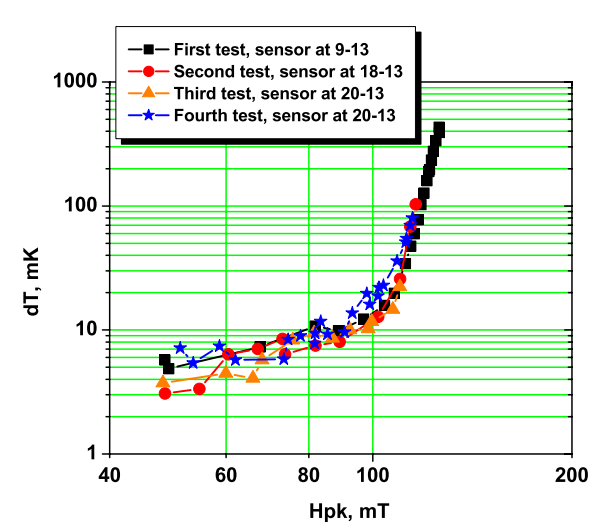

Figure 3: Thermometry data for high field Q-slope behavior.

In the third test the medium field Q-slope was strong, and it didn't show quadratic-type behavior. The quality factor was $3.6 \cdot 10^{10}$ at $\mathrm{H}_{\text {peak }}=32 \mathrm{mT}$. It corresponds to about $7.1 \mathrm{nOhm}$ of a mean surface resistance for this cavity. In this test the cavity performance degraded rapidly in the medium field Q-slope's region. The highest peak magnetic field was $110 \mathrm{mT}$, where the cavity was limited by a thermal breakdown. The thermal breakdown happened in the same place as in the previous test. The regions with high residual resistance were present, but covered only about 5 percent of the cavity surface, Fig. 5(triangles). There was a new dominating hot spot on the top half-cell. The temperature sensors in this region showed non-quadratic temperature growth at all fields, Fig. 6(triangles). This spot covered about 5 percent of cavity surface. The data from the rest of temperature sensors showed a quadratic field dependence, Fig. 3(triangles).

In the fourth test $R_{s}=8.09 \pm 0.10 \mathrm{nOhm}$ and $\gamma=$ $3.73 \pm 0.16$. The highest peak magnetic field was 110 $\mathrm{mT}$, where the cavity was limited by thermal breakdown. The thermal breakdown took place in the same place as before. The temperature map was still dominated by a hot spot at high fields on the top half-cell, which covered about 5 percent of the cavity surface. The temperature sensors in these regions showed non-quadratic behavior at all fields, Fig. 6(stars). The regions with high residual resistance 


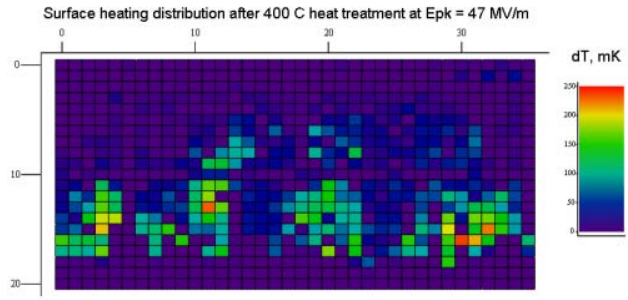

Figure 4: Temperature map for the second test at $\mathrm{H}_{\text {peak }}=117 \mathrm{mT}$.

were present and covered about 5 percent of the surface, Fig. 5(stars). The data from the rest of the temperature sensors showed quadratic field dependence, Fig. 3(stars).

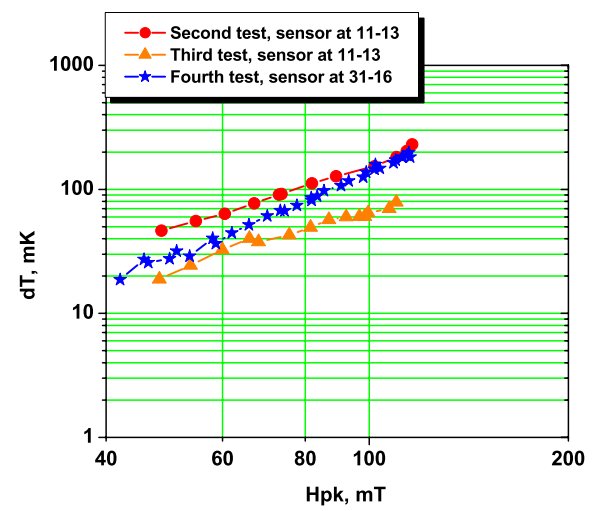

Figure 5: Thermometry data for behavior of new regions that appeared in the second test.

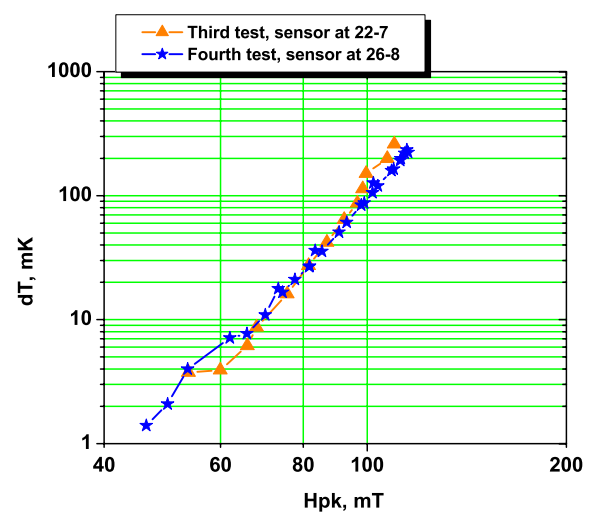

Figure 6: Thermometry data for behavior of the new region that appeared in the third test.

\section{DISCUSSION}

Our main interest was the high field Q-slope. It is known that the high field Q-slope can be removed or at least improved by a heat treatment at about $100^{\circ} \mathrm{C}$. It also was found that heat treatment at $150^{\circ} \mathrm{C}-180^{\circ} \mathrm{C}$ doesn't remove the high field Q-slope. To explain these experimental results a so-called "oxygen pollution model" was introduced [6]. The model suggests an existence of oxygenrich layer of niobium under the oxide, so called pollution layer. This oxygen-rich layer deteriorates RF properties of the superconductor. In this model the effect of a heat treatment is due to thermal diffusion of oxygen from the oxygen-rich layer into the bulk, thereby resulting in lower oxygen concentration in RF penetration depth and a better superconducting performance. In order to explain the effect of a $150^{\circ} \mathrm{C}-180^{\circ} \mathrm{C}$ heat treatment this model was modified by G.Ciovati [7]. Besides the initial pollution layer on the oxide-niobium interface, it was also proposed, that the destruction of the oxide during a heat treatment will resupply oxygen to the pollution layer. In this model an expression for oxygen concentration as a function of depth and its modification with heat treatment was proposed. We applied this modified model to our heat treatment in order to calculate the effect of our heat treatment conditions on the oxygen concentration in the London penetration depth. Our calculations show that in the London penetration depth the oxygen concentration after $400^{\circ} \mathrm{C}$ for 2 hours is lower than it is after $145^{\circ} \mathrm{C}$ for 45 hours. In the framework of the model the result means that a cavity's performance will improve after a high temperature heat treatment. Surprisingly, in our experiment after $400^{\circ} \mathrm{C}$ the performance of the high field Q-slope's regions remained practically unchanged at high fields, Fig. 1 and Fig. 3. So we suggest that the oxygen pollution layer is not responsible for the high field Q-slope. We will discuss a possible cause of new regions in another paper due to lack of space.

\section{CONCLUSION}

We have carried out a $400^{\circ} \mathrm{C}$ heat treatment on a $1500 \mathrm{MHz}$ elliptical single cell cavity prepared by vertical EP/flash BCP. We compared temperature maps before and after $400^{\circ} \mathrm{C}$ heat treatment, along with Q vs E curves. The surprising result is that $400^{\circ} \mathrm{C}$ heat treatment did not change the high field Q slope. Most thermometers at high field showed no substantial change due to $400{ }^{\circ} \mathrm{C}$ heat treatment. This result suggests that the high field Q-slope does not come from the oxide, nor the metal-oxide interface. However, in a few regions the temperature maps showed the development of new high resistance regions.

\section{REFERENCES}

[1] J.Knobloch, PhD Thesis, Cornell University, 1997.

[2] F.L. Palmer, IEEE TRANSACTIONS ON MAGNETICS, VOL. MAG-23, NO. 2, MARCH 1987.

[3] Q. Ma et al., JAP, Volume 96, Issue 12, pp. 7675-7680, 2004.

[4] R. Kirby et al., SLAC-TN-05-049, 2005.

[5] R.L.Geng et al., 12th Workshop on RF Superconductivity, 2005.

[6] H. Safa, 9th Workshop on RF Superconductivity, 1999.

[7] G. Ciovati, Applied Physics Letters 89,022507, 2006.

T07 Superconducting RF 